Tôhoku Math. Journ. 39 (1987), 61-69.

\title{
ON A METHOD TO CONSTRUCT ANALYTIC ACTIONS OF NON-COMPACT LIE GROUPS ON A SPHERE
}

\author{
FUICHI UCHIDA
}

(Received January 20, 1986)

0. Introduction. Let $M$ be a square matrix of degree $n$ with real coefficients, that is, $M \in M_{n}(\boldsymbol{R})$. We say that $M$ satisfies the outward transversality condition if

$$
\frac{d}{d t}\|\exp (t M) x\|>0 \text { for each } x \in \boldsymbol{R}_{0}^{n}=\boldsymbol{R}^{n}-\{0\} \text { and } t \in \boldsymbol{R} .
$$

In this case, there exists a unique real valued analytic function $\tau$ on $\boldsymbol{R}_{0}^{n}$ such that $\|\exp (\tau(x) M) x\|=1$, and hence we can define an analytic mapping $\pi^{\mu}$ of $\boldsymbol{R}_{0}^{n}$ onto the unit $(n-1)$-sphere $S^{n-1}$ by $\pi^{M}(x)=$ $\exp (\tau(x) M) x$.

Let $G$ be a Lie group, $\rho: G \rightarrow G L(n, \boldsymbol{R})$ a matricial representation, and $M$ a square matrix of degree $n$ with real coefficients satisfying the outward transversality condition. We can define an analytic mapping $\xi$ : $G \times S^{n-1} \rightarrow S^{n-1}$ by $\xi(g, x)=\pi^{\mu}(\rho(g) x)$, and we see that $\xi$ is an analytic $G$-action on $S^{n-1}$ if $\rho(g) M=M \rho(g)$ for any $g \in G$. We call $\xi$ a twisted linear action of $G$ on $S^{n-1}$ associated to the representation $\rho$. In particular, if $M$ is the identity matrix, we call $\xi$ a linear action of $G$ on $S^{n-1}$ associated to the representation $\rho$.

Let $G$ be a compact Lie group and $\rho: G \rightarrow G L(n, \boldsymbol{R})$ a matricial representation. Then we shall show that any twisted linear action of $G$ on $S^{n-1}$ associated to $\rho$ is equivariantly analytically diffeomorphic to the linear action of $G$ on $S^{n-1}$ associated to $\rho$. On the other hand, if $G$ is a non-compact Lie group, sometimes we can construct uncountably many topologically distinct twisted linear actions of $G$ associated to only one matricial representation (cf. $[4, \S 6])$. We shall study such an example in the final section.

\section{Outward transversality condition.}

1.1. Let $u=\left(u_{i}\right)$ and $v=\left(v_{i}\right)$ be vectors in $\boldsymbol{R}^{n}$. As usual, we denote their inner product by $u \cdot v=\sum_{i} u_{i} v_{i}$ and the length of $u$ by $\|u\|=\sqrt{u \cdot u}$.

LEMmA 1.1. Let $M \in M_{n}(\boldsymbol{R})$ and assume that $M$ satisfies the outward transversality condition. Then, (i) 


$$
\lim _{t \rightarrow+\infty}\|\exp (t M) x\|=+\infty \text { and } \lim _{t \rightarrow-\infty}\|\exp (t M) x\|=0
$$

for each $x \in \boldsymbol{R}_{0}^{n}$, and (ii) there exists a unique real valued analytic function $\tau$ on $\boldsymbol{R}_{0}^{n}$ such that $\|\exp (\tau(x) M) x\|=1$ for each $x \in \boldsymbol{R}_{0}^{n}$.

Proof. Put $f(t ; x)=\|\exp (t M) x\|$. Because $M$ satisfies the outward transversality condition, there exists $\varepsilon>0$ satisfying $f^{\prime}(0 ; x) \geqq \varepsilon$ for $x \in S^{n-1}$. Then

$$
\begin{aligned}
f^{\prime}(t ; x) & =f^{\prime}(0 ; \exp (t M) x) \\
& =\|\exp (t M) x\| \cdot f^{\prime}\left(0 ;\|\exp (t M) x\|^{-1} \exp (t M) x\right) \geqq \varepsilon \cdot f(t ; x)
\end{aligned}
$$

for each $x \in \boldsymbol{R}_{0}^{n}$ and $t \in \boldsymbol{R}$. Hence we obtain

$$
\frac{d}{d t} \log f(t ; x) \geqq \varepsilon \quad \text { for } \quad x \in \boldsymbol{R}_{0}^{n}, t \in \boldsymbol{R} .
$$

Integrating both sides of the inequality, we obtain

$$
\begin{array}{ll}
\|\exp (t M) x\| \geqq\|x\| \exp (\varepsilon t) & \text { for } t>0, \\
\|\exp (t M) x\| \leqq\|x\| \exp (\varepsilon t) & \text { for } t<0 .
\end{array}
$$

The condition (i) follows from these inequalities. The function $f(t ; x)$ is strictly monotone by the assumption on $M$. Thus the condition (i) assures the unique existence of $\tau: \boldsymbol{R}_{0}^{n} \rightarrow \boldsymbol{R}$ satisfying $\|\exp (\tau(x) M) x\|=1$ for each $x \in \boldsymbol{R}_{0}^{n}$. On the other hand, we see that $\tau$ is analytic, applying the implicit function theorem to the analytic function $(x, t) \rightarrow\|\exp (t M) x\|$, because $M$ satisfies the outward transversality condition.

q.e.d.

REMARK. Conversely, we can prove that the conditions (i), (ii) are sufficient for $M$ to satisfy the outward transversality condition.

By this lemma, we can define an analytic mapping $\pi^{M}: \boldsymbol{R}_{0}^{n} \rightarrow S^{n-1}$ by $\pi^{M}(x)=\exp (\tau(x) M) x$, if $M$ satisfies the outward transversality condition.

1.2. Let $G$ be a Lie group and $\rho: G \rightarrow G L(n, \boldsymbol{R})$ a matricial representation. Denote by $\operatorname{End}_{G}(\rho)$ the set of all matrices $X \in M_{n}(\boldsymbol{R})$ satisfying $X \rho(g)=\rho(g) X$ for $g \in G$. The set $G L(n, \boldsymbol{R}) \cap \operatorname{End}_{G}(\rho)$ is denoted by $\operatorname{Aut}_{G}(\rho)$. If $M \in \operatorname{End}_{G}(\rho)$ and $M$ satisfies the outward transversality condition, we call $(\rho, M)$ a TC-pair of degree $n$. In this case, we can define an analytic mapping $\xi: G \times S^{n-1} \rightarrow S^{n-1}$ by $\xi(g, x)=\pi^{\mu}(\rho(g) x)$ and we see easily that $\xi$ is an action of $G$ on $S^{n-1}$. We call $\xi$ a twisted linear action of $G$ on $S^{n-1}$ determined by the TC-pair $(\rho, M)$. In particular, if $M$ is the identity matrix $I_{n}$, we call $\xi$ a linear action of $G$ on $S^{n-1}$ associated to $\rho$.

Let $(\rho, M)$ and $(\sigma, N)$ be TC-pairs of degree $n$. We say that $(\rho, M)$ 
is equivalent to $(\sigma, N)$ if there exist $A \in G L(n, \boldsymbol{R})$ and a positive real number $c$ such that $c N=A M A^{-1}$ and $\sigma(g) A=A \rho(g)$ for any $g \in G$.

LEMMA 1.2. If $(\rho, M)$ and $(\sigma, N)$ are equivalent as TC-pairs, then the twisted linear action of $G$ on a sphere determined by $(\rho, M)$ is equivariantly analytically diffeomorphic to the one determined by $(\sigma, N)$.

Proof. It is easy to see that the twisted linear action of $G$ determined by $(\sigma, c N)$ coincides with the one determined by $(\sigma, N)$ for any positive real number $c$. So we assume that there exists $A \in G L(n, R)$ such that

$$
N=A M A^{-1} \text { and } \sigma(g) A=A \rho(g) \text { for any } g \in G .
$$

Define analytic mappings $h_{A}, k_{A}$ of $S^{n-1}$ into itself by $h_{A}(x)=\pi^{N}(A x)$ and $k_{A}(y)=\pi^{M}\left(A^{-1} y\right)$. Then we see that the composites $h_{A} k_{A}$ and $k_{A} h_{A}$ are the identity mappings on $S^{n-1}$ by the condition $N=A M A^{-1}$, and hence $h_{A}: S^{n-1} \rightarrow S^{n-1}$ is an analytic diffeomorphism. In addition, we see that

$$
h_{A}\left(\pi^{\mu}(\rho(g) x)\right)=\pi^{N}\left(\sigma(g) h_{A}(x)\right) \quad \text { for } \quad g \in G, x \in S^{n-1}
$$

by the condition $(*)$.

q.e.d.

Lemma 1.3. Let $M=\left(m_{i j}\right)$ be a square matrix of degree $n$ with real coefficients. Then $M$ satisfies the outward transversality condition if and only if the quadratic form

$$
x \cdot M x=\sum_{i, j} m_{i j} x_{i} x_{j}
$$

is positive definite.

Proof. The result follows immediately from the equality:

$$
\begin{aligned}
2(\exp (t M) x) \cdot(M \exp (t M) x) & =\frac{d}{d t}\|\exp (t M) x\|^{2} \\
& =2\|\exp (t M) x\| \frac{d}{d t}\|\exp (t M) x\| \cdot \quad \text { q.e.d. }
\end{aligned}
$$

\section{Positive definite quadratic forms.}

2.1. Let $\boldsymbol{F}$ denote the field of real numbers $\boldsymbol{R}$, complex numbers $\boldsymbol{C}$, or quaternions $\boldsymbol{Q}$. As usual, let $M_{n}(\boldsymbol{F})$ denote the set of all matrices of degree $n$ with coefficients in $\boldsymbol{F}$, and let $G L(n, \boldsymbol{F})$ denote the general linear group consisting of regular matrices in $M_{n}(\boldsymbol{F})$. Let $u=\left(u_{i}\right)$ and $v=\left(v_{i}\right)$ be vectors in $\boldsymbol{F}^{n}$, the $n$-dimensional cartesian space over the field $\boldsymbol{F}$. As usual, we define their inner product by $u \cdot v=\sum_{i} \bar{u}_{i} v_{i}$, and the length of $u$ to be the number $\|u\|=\sqrt{u \cdot u}$.

We define $\iota_{1}: M_{n}(\boldsymbol{C}) \rightarrow M_{2 n}(\boldsymbol{R})$ and $\iota_{2}: M_{n}(\boldsymbol{Q}) \rightarrow M_{2 n}(\boldsymbol{C})$ by 


$$
\iota_{1}(A+i B)=\left(\begin{array}{rr}
A & -B \\
B & A
\end{array}\right) \quad \text { and } \quad \iota_{2}(C+j D)=\left(\begin{array}{lr}
C & -\bar{D} \\
D & \bar{C}
\end{array}\right)
$$

where $A, B \in M_{n}(\boldsymbol{R})$ and $C, D \in M_{n}(\boldsymbol{C})$. Then we see that $\iota_{1}$ and $\iota_{2}$ are injective ring homomorphisms. We define $\iota: M_{n}(\boldsymbol{F}) \rightarrow M_{k n}(\boldsymbol{R})$ by $(k, \iota)=$ (1, id.), $\left(2, \iota_{1}\right)$ and $\left(4, \iota_{1} \iota_{2}\right)$ for $\boldsymbol{F}=\boldsymbol{R}, \boldsymbol{C}$ and $\boldsymbol{Q}$, respectively.

If $u=x+i y \in \boldsymbol{C}^{n}$ and $v=z+j w \in \boldsymbol{Q}^{n}$, we assign to $u$ and $v$ the vectors $u^{\prime}=\left(\begin{array}{l}x \\ y\end{array}\right) \in R^{2 n}$ and $v^{\prime}=\left(\begin{array}{c}z \\ w\end{array}\right) \in C^{2 n}$, respectively. Moreover, we assign to $v \in \boldsymbol{Q}^{n}$ the vector $v^{\prime \prime}=\left(v^{\prime}\right)^{\prime} \in \boldsymbol{R}^{4 n}$. We have the following.

$$
\begin{aligned}
& \operatorname{Re}(u \cdot X u)=u^{\prime} \cdot \iota(X) u^{\prime} \text { for } X \in M_{n}(\boldsymbol{C}), u \in \boldsymbol{C}^{n}, \\
& \operatorname{Re}(v \cdot X v)=v^{\prime \prime} \cdot \iota(X) v^{\prime \prime} \quad \text { for } \quad X \in M_{n}(\boldsymbol{Q}), v \in \boldsymbol{Q}^{n},
\end{aligned}
$$

where $\operatorname{Re}()$ denotes the real part.

LEMMA 2.2. Let $X \in M_{n}(\boldsymbol{F})$ and assume that all the eigenvalues of $\iota(X)$ have positive real parts. Then there exists $P \in G L(n, F)$ such that $\operatorname{Re}\left(u \cdot P X P^{-1} u\right)>0$ for $u \in F^{n}-\{0\}$.

Proof. Notice that if $\lambda_{1}, \cdots, \lambda_{n}$ are the eigenvalues of $X \in M_{n}(\boldsymbol{C})$ then $\lambda_{1}, \cdots, \lambda_{n}, \bar{\lambda}_{1}, \cdots, \bar{\lambda}_{n}$ are the eigenvalues of $\iota_{1}(X)$, and hence the result for $\boldsymbol{F}=\boldsymbol{R}$ and $\boldsymbol{C}$ is proved essentially as in the case of Lyapunov functions in $[2, \S 22.3-\S 22.5]$. Here we shall prove the result for $\boldsymbol{F}=\boldsymbol{Q}$ by the same method for completeness. Let $X \in M_{n}(\boldsymbol{Q})$ and assume that all the eigenvalues of $\iota_{2}(X)$ have positive real parts. Let $\lambda$ be an eigenvalue of $\iota_{2}(X)$. Then there exists a unit vector $v \in \boldsymbol{Q}^{n}$ such that $\iota_{2}(X) v^{\prime}=v^{\prime} \lambda$; hence we have $X v=v \lambda$. There exists $P_{0} \in S p(n)$ such that $P_{0}^{-1} e_{1}=v$ (cf. [3, ch. I, §VII]). Then $P_{0} X P_{0}^{-1} e_{1}=e_{1} \lambda$; in other words,

$$
P_{0} X P_{0}^{-1}=\left(\begin{array}{cc}
\lambda & \\
0 & \\
\vdots & \\
0 &
\end{array}\right) \text {. }
$$

By induction on $n$, we have an element $P_{1} \in S p(n)$ such that

$$
P_{1} X P_{1}^{-1}=\left(\begin{array}{lll}
\lambda_{1} & & x_{i j} \\
& \ddots & \\
0 & & \lambda_{n}
\end{array}\right)
$$

where $\lambda_{1}, \cdots, \lambda_{n}$ are complex numbers and $x_{i j}$ 's are quaternions. Then we see that $\lambda_{1}, \cdots, \lambda_{n}, \bar{\lambda}_{1}, \cdots, \bar{\lambda}_{n}$ are the eigenvalues of $\iota_{2}(X)$. We define positive real numbers $a, b$ by

$$
a=\min _{i} \operatorname{Re}\left(\lambda_{i}\right), \quad b=n(n-1)\left(a+\max _{i<j}\left|x_{i j}\right|\right) a^{-1} .
$$


Let $P_{2}$ be the diagonal matrix with diagonal entries $b, b^{2}, \cdots, b^{n}$, and put $P=P_{2} P_{1}$. Then we have

$$
P X P^{-1}=\left(\begin{array}{lll}
\lambda_{1} & & a_{i j} \\
& \ddots & \\
0 & & \lambda_{n}
\end{array}\right),
$$

where $a_{i j}=b^{i-j} x_{i j}$. We shall show that $P$ is a desired matrix. We have

$$
u \cdot P X P^{-1} u=\sum_{i} \bar{u}_{i} \lambda_{i} u_{i}+\sum_{i<j} \bar{u}_{i} a_{i j} u_{j}
$$

for $u=\left(u_{i}\right) \in \boldsymbol{Q}^{n}$, and hence

$$
\operatorname{Re}\left(u \cdot P X P^{-1} u\right)=\sum_{i} \operatorname{Re}\left(\lambda_{i}\right)\left|u_{i}\right|^{2}+\sum_{i<j} \operatorname{Re}\left(\bar{u}_{i} a_{i j} u_{j}\right) .
$$

Therefore

$$
\left.\left|\operatorname{Re}\left(u \cdot P X P^{-1} u\right)-\sum_{i} \operatorname{Re}\left(\lambda_{i}\right)\right| u_{i}\right|^{2}\left|\leqq \sum_{i<j}\right| a_{i j} \mid\|u\|^{2} \leqq \frac{a}{2}\|u\|^{2},
$$

because $\left|a_{i j}\right| \leqq a / n(n-1)$ for $i<j$. Consequently, we obtain

$$
\operatorname{Re}\left(u \cdot P X P^{-1} u\right) \geqq \sum_{i} \operatorname{Re}\left(\lambda_{i}\right)\left|u_{i}\right|^{2}-\frac{a}{2}\|u\|^{2} \geqq \frac{a}{2}\|u\|^{2}>0
$$

for $u \in Q^{n}-\{0\}$.

q.e.d.

2.2. Next we shall show the following.

THEOREM 2.3. The following three conditions are equivalent for $X \in M_{n}(\boldsymbol{R})$.

(1) All the eigenvalues of $X$ have positive real parts.

(2) There exists $P \in G L(n, R)$ such that the quadratic form $u \cdot P X P^{-1} u$ is positive definite.

(3) $\lim _{t \rightarrow+\infty}\|\exp (t X) u\|=+\infty, \quad \lim _{t \rightarrow-\infty}\|\exp (t X) u\|=0 \quad$ for $u \in \boldsymbol{R}_{0}^{n}$.

Proof. The condition (1) implies (2) by Lemma 2.2. If $A \in G L(n, \boldsymbol{R})$ and $x \in \boldsymbol{R}^{n}$, then we have

$$
\left\|A^{-1}\right\|^{-1}\|x\| \leqq\|A x\| \leqq\|A\|\|x\|,
$$

where $\|A\|^{2}=\operatorname{trace}^{t} A A$. In particular,

$$
\|P\|^{-1}\left\|\exp \left(t P X P^{-1}\right) P u\right\| \leqq\|\exp (t X) u\| \leqq\left\|P^{-1}\right\|\left\|\exp \left(t P X P^{-1}\right) P u\right\|
$$

for $X \in M_{n}(\boldsymbol{R}), \quad P \in G L(n, \boldsymbol{R})$ and $u \in \boldsymbol{R}^{n}$. Therefore Lemma 1.1 and Lemma 1.3 assure that the condition (2) implies (3). Finally, we shall show that the condition (3) implies (1). Let $\lambda=a+i b$ be an eigenvalue 
of $X$, and let $z=x+i y$ be a unit eigenvector of $X$ in $C^{n}$ belonging to $\lambda$. Then

$$
\|\exp (t X) x\|^{2}+\|\exp (t X) y\|^{2}=e^{2 t a}\|z\|^{2}=e^{2 t a} .
$$

The condition (3) for the matrix $X$ implies $a>0$.

q.e.d.

3. Twisted linear actions for compact Lie groups. Let $\alpha=\left(a_{i j}\right)$ and $\beta=\left(b_{k l}\right)$ be matrices of degrees $p$ and $q$, respectively. We denote by $\alpha \oplus \beta$ the matrix $\left(\begin{array}{ll}\alpha & 0 \\ 0 & \beta\end{array}\right)$ of degree $p+q$, and denote by $\alpha \otimes \beta$ the Kronecker product, that is, the matrix $\left(c_{r s}\right)$ of degree $p q$ whose coefficients are given by $c_{r s}=a_{i j} b_{k l}$ for $r=i+p(k-1), s=j+p(l-1)$.

Let $\rho: G \rightarrow G L(n, \boldsymbol{R})$ be a matricial representation of a Lie group $G$. We say that $\rho$ is in standard form, if there exist irreducible representations $\rho_{i}: G \rightarrow G L\left(n_{i}, \boldsymbol{F}_{i}\right)(i=1,2, \cdots, r)$ such that

$$
\begin{aligned}
& \rho=\left(\rho_{1} \otimes I_{k_{1}}\right) \oplus \cdots \oplus\left(\rho_{r} \otimes I_{k_{r}}\right), \\
& \operatorname{End}_{G}(\rho)=\left(I_{n_{1}} \otimes M_{k_{1}}\left(\boldsymbol{F}_{1}\right)\right) \oplus \cdots \oplus\left(I_{n_{r}} \otimes M_{k_{r}}\left(\boldsymbol{F}_{r}\right)\right),
\end{aligned}
$$

where $\boldsymbol{F}_{i}=\boldsymbol{R}, \boldsymbol{C}$ or $\boldsymbol{Q}$. It is well known that any matricial representation of a compact Lie group is equivalent to one in standard from (cf. $[1$, ch. 3$],[3$, ch. VI]).

LEMMA 3.2. Let $\rho$ be a matricial representation in standard form of a Lie group G. Let $X \in \operatorname{End}_{G}(\rho)$ and assume that all the eigenvalues of $X$ have positive real parts. Then there exists $P \in \operatorname{Aut}_{G}(\rho)$ such that $P X P^{-1}$ satisfies the outward transversality condition.

Proof. The result follows immediately from (3.1), (2.1), Lemma 2.2 and Lemma 1.3.

q.e.d.

REMARK. If $\rho: G \rightarrow G L(n, \boldsymbol{R})$ is an irreducible representation which has no complex structure, then the linear action is the unique twisted linear action of $G$ on $S^{n-1}$ associated to $\rho$.

THEOREM 3.3. Let $G$ be a compact Lie group and $\rho: G \rightarrow G L(n, \boldsymbol{R})$ a matricial representation. Then any twisted linear action of $G$ on $S^{n-1}$ associated to $\rho$ is equivariantly analytically diffeomorphic to the linear action of $G$ on $S^{n-1}$ associated to $\rho$.

Proof. Let $M \in \operatorname{End}_{G}(\rho)$ and assume that $M$ satisfies the outward transversality conditition. We shall show that the twisted linear action of $G$ on $S^{n-1}$ determined by the TC-pair $(\rho, M)$ is equivariantly analytically diffeomorphic to the linear action of $G$ on $S^{n-1}$ associated to $\rho$. Since $G$ is compact, there are $P_{1} \in G L(n, \boldsymbol{R})$ and an orthogonal representation 
$\sigma$ in standard form satisfying $\sigma(g) P_{1}=P_{1} \rho(g)$ for any $g \in G$. Then $P_{1} M P_{1}^{-1} \in$ $\operatorname{End}_{G}(\sigma)$ and all the eigenvalues of $P_{1} M P_{1}^{-1}$ have positive real parts. Thus there exists $P_{2} \in \mathrm{Aut}_{G}(\sigma)$ such that $P_{2} P_{1} M P_{1}^{-1} P_{2}^{-1}$ satisfies the outward transversality condition by Lemma 3.2. Let $P=P_{2} P_{1}$ and $N=P M P^{-1}$. Define analytic diffeomorphisms $h_{P}, k_{P}$ of $S^{n-1}$ onto itself by $h_{P}(x)=\pi^{N}(P x)$ and $k_{P}(x)=\pi^{I_{n}}\left(P^{-1} x\right)$. As in the proof of Lemma 1.2, we see that $h_{P}$ is an equivariant analytic diffeomorphism from $S^{n-1}$ with the twisted linear action determined by the TC-pair $(\rho, M)$ onto $S^{n-1}$ with the one determined by the TC-pair $(\sigma, N)$, and $k_{P}$ is an equivariant analytic diffeomorphism from $S^{n-1}$ with the linear action associated to $\sigma$ onto $S^{n-1}$ with the one associated to $\rho$. Since $\sigma$ is an orthogonal representation, the twisted linear action of $G$ on $S^{n-1}$ determined by the TC-pair $(\sigma, N)$ coincides with the linear action associated to $\sigma$. Therefore the composite $k_{P} h_{P}$ is an equivariant analytic diffeomorphism from $S^{n-1}$ with the twisted linear action determined by the TC-pair $(\rho, M)$ onto $S^{n-1}$ with the linear action associated to $\rho$.

q.e.d.

4. Typical example. Here we shall study twisted linear actions of $G=S L(n, \boldsymbol{R})$ on $S^{2 n-1}$ associated to $\rho_{n} \otimes I_{2}$, where $\rho_{n}: S L(n, \boldsymbol{R}) \rightarrow G L(n, \boldsymbol{R})$ is the natural inclusion. We have $\operatorname{End}_{G}\left(\rho_{n} \otimes I_{2}\right)=I_{n} \otimes M_{2}(R)$. Let $e_{1}, \cdots, e_{n}$ be the standard base of $\boldsymbol{R}^{n}$.

LEMMA 4.1. Let $u, v$ be vectors in $\boldsymbol{R}^{n}$. If $u$, $v$ are linearly independent and $n \geqq 3$, then there exists $P \in S L(n, R)$ such that $P u=(1 / \sqrt{2}) e_{1}$ and $P v=(1 / \sqrt{\mathbf{2}}) e_{2}$.

Proof. Since $u, v$ are linearly independent, there exists $P_{1} \in S O(n)$ such that $P_{1} u=p e_{1}$ and $P_{1} v=q e_{1}+r e_{2}$ for some real numbers $p, q, r$ satisfying $p r \neq 0$. Next, since $n \geqq 3$, there exists $P_{2} \in S L(n, \boldsymbol{R})$ such that $P_{2} e_{1}=(1 / p \sqrt{2}) e_{1}$ and $P_{2} e_{2}=(-q / p r \sqrt{2}) e_{1}+(1 / r V \overline{2}) e_{2}$. We are done by letting $P=P_{2} P_{1}$.

q.e.d.

By this lemma, we see that the orbit through $(1 / \sqrt{2})\left(e_{1} \oplus e_{2}\right)$ is open and dense in $S^{2 n-1}$ for any twisted linear action of $S L(n, \boldsymbol{R})$ associated to $\rho_{n} \otimes I_{2}$, because the orbit consists of all $u \oplus v \in S^{2 n-1}$ such that $u, v$ are linearly independent.

Let $M \in M_{2}(\boldsymbol{R})$ and assume that $M$ satisfies the outward transversality condition. Then $\left(\rho_{n} \otimes I_{2}, I_{n} \otimes M\right)$ is a TC-pair. In fact, $I_{n} \otimes M$ satisfies the outward transversality condition if and only if $M$ satisfies the condition. Denote by $I^{n}(M)$ the isotropy group at $(1 / \sqrt{2})\left(e_{1} \oplus e_{2}\right)$ with respect to the twisted linear action of $S L(n, \boldsymbol{R})$ on $S^{2 n-1}$ determined by the TCpair $\left(\rho_{n} \otimes I_{2}, I_{n} \otimes M\right)$. We see easily $X \in I^{n}(M)$ if and only if 


$$
X=\left(\begin{array}{c|c}
{ }^{t} \exp (\theta M) & * \\
\hline 0 & *
\end{array}\right)
$$

for some $\theta \in \boldsymbol{R}$.

LEMMA 4.3. With respect to the natural action of $I^{n}(M)$ on $\boldsymbol{R}^{n}$ as a subgroup of $S L(n, \boldsymbol{R})$, the subspace spanned by $\left\{e_{1}, e_{2}\right\}$ is the unique invariant 2-dimensional linear subspace.

Proof. Let $V$ be an invariant linear subspace of $\boldsymbol{R}^{n}$, and assume that $V$ contains a vector which is not a linear combination of $e_{1}, e_{2}$. Then we see that $V$ contains $e_{1}$ and $e_{2}$, because any matrix of the form

$$
\left(\begin{array}{ll}
I_{2} & * \\
0 & I_{n-2}
\end{array}\right)
$$

is contained in $I^{n}(M)$.

q.e.d.

Let $M, N \in M_{2}(\boldsymbol{R})$. We say that $M$ is similar to $N$ up to positive scalar multiplication, if there exist $P \in G L(2, R)$ and a positive real number $c$ such that $c N=P M P^{-1}$.

LEMMA 4.4. Let $M, N \in M_{2}(\boldsymbol{R})$ and assume that $M, N$ satisfy the outward transversality condition. If $n \geqq 3$, then the following two conditions are equivalent.

(1) $M$ is similar to $N$ up to positive scalar multiplication.

(2) $I^{n}(M)$ and $I^{n}(N)$ are conjugate in $S L(n, R)$.

Proof. By Lemma 1.2, we see that the condition (1) implies (2). Now we shall show that the condition (2) implies (1). Assume that there exists $A \in S L(n, R)$ such that $I^{n}(N)=A I^{n}(M) A^{-1}$. Then, by Lemma 4.3, we see that the subspace spanned by $\left\{e_{1}, e_{2}\right\}$ is $A$-invariant, and hence $A=\left(\begin{array}{ll}B & * \\ 0 & *\end{array}\right)$ for some $B \in G L(2, \boldsymbol{R})$. By (4.2), we obtain $c N={ }^{t} B^{-1} M^{t} B$ for a real number $c$. We see $c>0$, because $M, N$ satisfy the outward transversality condition.

q.e.d.

By this lemma, if $\left(\rho_{n} \otimes I_{2}, I_{n} \otimes M\right)$ and $\left(\rho_{n} \otimes I_{2}, I_{n} \otimes N\right)$ are not equivalent as TC-pairs, then there is no equivariant homeomorphism from $S^{2 n-1}$ with the twisted linear action of $S L(n, \boldsymbol{R})$ determined by the TCpair $\left(\rho_{n} \otimes I_{2}, I_{n} \otimes M\right)$ onto $S^{2 n-1}$ with the one determined by the TC-pair $\left(\rho_{n} \otimes I_{2}, I_{n} \otimes N\right)$.

We see easily the following. Let $M=\left(\begin{array}{ll}a & b \\ c & d\end{array}\right)$. Then $M$ satisfies the outward transversality condition if and only if $a>0$ and $4 a d-(b+c)^{2}>0$, by Lemma 1.3. The following matrices satisfy the outward transversality 
condition.

$$
\left(\begin{array}{ll}
1 & 1 \\
0 & 1
\end{array}\right), \quad\left(\begin{array}{ll}
1 & 0 \\
0 & x
\end{array}\right) \quad(0<x \leqq 1), \quad\left(\begin{array}{rr}
1 & y \\
-y & 1
\end{array}\right) \quad(y>0) .
$$

Moreover, no two of them are similar up to positive scalar multiplication, and any matrix of degree 2 satisfying the outward transversality condition is similar to one of the above matrices up to positive scalar multiplication.

\section{REFERENCES}

[1] J.F. AdAms, Lectures on Lie Groups, Benjamin Inc., 1969.

[2] V.I. ARNold, Ordinary Differential Equations (English translation), MIT Press, 1978.

[3] C. Chevalley, Theory of Lie Groups I, Princeton Univ. Press, 1946.

[4] F. UchidA, Real analytic actions of complex symplectic groups and other classical Lie groups on spheres, J. Math. Soc. Japan, to appear.

Department of Mathematics

Faculty of Science

YaMAGATA UNIVERSITY

YaMAGATA 990 , JAPAN 
